\title{
Nonlinear Dynamic Identification of Beams Resting on Nonlinear Viscoelastic Foundations Based on the Time-Delayed Transfer Entropy and Improved Surrogate Data Algorithm
}

\author{
Guohua Liu $\mathbb{D}^{1},{ }^{1}$ Changpeng Ye $\mathbb{D}^{1},{ }^{1} \mathrm{Xu}$ Liang $\mathbb{D}^{2},{ }^{2}$ Zhongkai Xie, $^{3}$ and Ziyuan Yu $\mathbb{D}^{1}$ \\ ${ }^{1}$ College of Civil Engineering and Architecture, Zhejiang University, Hangzhou 310058, China \\ ${ }^{2}$ Ocean College, Zhejiang University, Zhoushan 316021, China \\ ${ }^{3}$ Zhejiang Institute of Hydraulics \& Estuary, Hangzhou 310020, China \\ Correspondence should be addressed to Xu Liang; liangxu@zju.edu.cn
}

Received 24 May 2018; Accepted 6 September 2018; Published 25 September 2018

Academic Editor: Carlo Rainieri

Copyright (C) 2018 Guohua Liu et al. This is an open access article distributed under the Creative Commons Attribution License, which permits unrestricted use, distribution, and reproduction in any medium, provided the original work is properly cited.

\begin{abstract}
In this work, the transfer entropy and surrogate data algorithm were introduced to identify the nonlinearity level of the system by using a numerical solution of nonlinear response of beams. A homogeneous Euler-Bernoulli beam was subjected to a timevarying concentrated load and resting on a nonlinear foundation. The Galerkin method was applied to discretize the dimensionless differential governing equation of the forced vibration, and then the fourth-order Runge-Kutta method was used to obtain the timehistory response of the lateral displacement. In order to simulate different nonlinearity levels, different ratios between nonlinear parameters and linear parameters of foundation, as well as different Young's moduli, were used. A nonlinearity index was proposed. In the case of different nonlinearity levels, the nonlinearity index was used to analyze the difference between the transfer entropy calculated from the original data and the transfer entropy calculated from the surrogate data. By comparing and analyzing the nonlinearity index values under different ratios, it was found that the nonlinearity index values generally increased with the increase of the ratio and the sum of nonlinearity index values had a positive correlation with the ratio. By comparing the nonlinearity index values of the transfer entropy results of beams with different Young's moduli, it was found that the sum of the nonlinearity index values generally decreased with the increase of Young's modulus. The numerical results demonstrate that the present approach could effectively quantify the nonlinearity in the response of a beam resting on a nonlinear foundation.
\end{abstract}

\section{Introduction}

The major differences between heavy-haul railways and general railways are the high traffic volumes and high traffic density, so that the load intensity and fatigue effect of the railway subgrade are raised. In particular, after the speed has been enhanced, there is increasingly more damage occurring in the existing railway subgrade. As a result, the railway track is not smooth, which aggravates the dynamic effect between the train and the track and seriously influences the transport capacity of the railway. In structural engineering, damage is often considered to be the introduction of a nonlinearity into a structure or structural component [1]. Therefore, common defects in heavy-haul railway subgrades can be introduced into the subgrade as nonlinearity, so that the track on heavy-haul railway subgrade with defects is considered as the beam on a nonlinear foundation.

Recently, scholars both at home and abroad have studied the beam on nonlinear viscoelastic foundations subjected to time-dependent loads. Kargarnovin et al. [2] used a perturbation method in conjunction with a complex Fourier transformation to study the response of infinite beams supported by nonlinear viscoelastic foundations subjected to harmonic moving loads. A. D. Senalp et al. [3] investigated the dynamic response of a simply supported, finite length Euler-Bernoulli beam with uniform cross-section resting on a linear and nonlinear viscoelastic foundation subjected to a moving concentrated load and utilized the Galerkin method to solve the governing equations of motion. Ansari et al. [4] used the Galerkin method and the Multiple Scales Method (MSM) to 
study the transverse vibration of a finite Euler-Bernoulli beam supported by nonlinear viscoelastic foundations. S.M. Abdelghany et al. [5] used the Galerkin with Runge-Kutta methods to investigate the dynamic response of nonuniform EulerBernoulli simply supported beam subjected to moving load.

The subgrade structure is hidden, and an internal change in the subgrade structure is a gradual and complex process that is not easy to visually detect. The quality of the subgrade structure directly affects the working status and service life of the track. Therefore, it is necessary to identify the degree of nonlinearity of the subgrade to determine whether the foundation is damaged or not and prevent accidents from occurring.

With deepening research on the mechanism of the nonlinear vibration response in structural dynamics, a variety of different nonlinear system identification methods have emerged. Based on the dynamic equilibrium equation of the dynamical system, Masri S F et al. [6-9] presented a wellknown nonlinear identification theory called the restoring force surface method and successfully applied it to the identification of a nonlinear multidegree of freedom system. Mohammad et al. [10] presented a direct parameter identification method based entirely on excitation and response, which effectively identified parameters such as mass, stiffness, and damping of linear and nonlinear systems. Based on the Hilbert transform, Huang et al. [11] presented a new data processing and analysis method called the HilbertHuang transform. This method did not need to impose any restrictions on the data and can accurately represent the most essential features of the linear stationary signals and nonlinear nonstationary signals.

The identification techniques of the damage-induced nonlinearities in structures have recently combined the use of information theoretic-based methods. As a generalized information-based approach, the transfer entropy examines the structural dynamics on a probabilistic description from the perspective of information transfer between different locations on a structure and uses conditional relationships to determine the degree and direction of information flow [12]. The transfer entropy considers not only the second-order correlations in the data but rather the higher order nonlinearity correlations over the entire probability distribution of the data. The transfer entropy does not require a particular nonlinearity model or preprocessing of the response time series and is suitable for any type of stationary excitation [13]. By using a numerical analysis method, Xie et al. [14] concluded that the transfer entropy was effectively used to quantify and locate the damage in concrete structures. Sun et al. [15] proposed an identification method of the transmission path and transmission direction of vibration information of hydropower stations based on the transfer entropy. To solve the difficulty of obtaining linear baseline data in practical engineering, the transfer entropy combined with the surrogate data algorithm has been adopted to produce statistically significant results for nonlinear identification applications. The first step of the method is often to construct surrogate data sets from the original structural response data and then the time-delayed transfer entropy measures on both the original data and the surrogate data are computed [13].

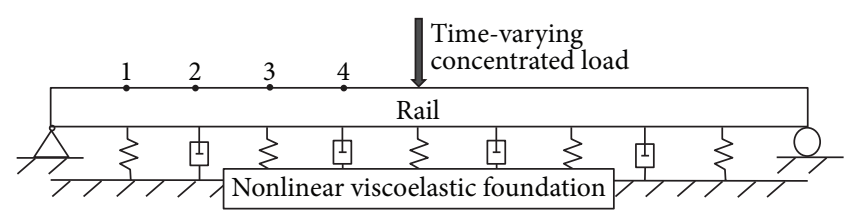

FIgURE 1: A homogeneous Euler-Bernoulli beam on a nonlinear viscoelastic foundation under a time-dependent load.

Finally, the differences between the two kinds of results are compared. Nichols et al. [16] used the time-delayed transfer entropy combined with the surrogate data algorithm to diagnose effectively the presence of impact damage in a thick composite sandwich plate.

Here, we propose a method based on the time-delayed transfer entropy and surrogate data algorithm to identify the nonlinearity of a beam on a nonlinear viscoelastic foundation. By establishing a nonlinearity index to describe the nonlinearity of the system quantitatively, the transfer entropy is effectively applied to the nonlinear identification of the dynamic system. The dynamic response of a homogeneous Euler-Bernoulli simply supported beam that is rested on a nonlinear viscoelastic foundation and subjected to a timedependent load is calculated with the Galerkin method combined with the fourth-order Runge-Kutta method in Section 2. In Section 3, the time-delayed transfer entropy and improved surrogate data algorithm are introduced. Since the computation of transfer entropy involves the evaluation of multiple probability densities, a kernel density estimation algorithm is introduced. To remove the dependence on the initial state of the structure, the surrogate data algorithm is introduced at the same time. At the end of Section 3, we also construct a nonlinearity index to quantitatively describe the nonlinearity degree of the structure. Section 4 uses the method introduced in Section 2 to obtain the time response results of the lateral displacement of the beam under different nonlinearity levels. The time-delayed transfer entropy is computed on both the original data and the surrogate data of the time response results. Finally, the nonlinearity index results are calculated, and the calculation results are analyzed to prove the applicability of the method.

\section{Calculation Method of the Dynamic Response of an Euler-Bernoulli Beam Rested on a Nonlinear Viscoelastic Foundation}

2.1. Statement of the Problem. A homogeneous Euler-Bernoulli beam of length $\mathrm{L}$ on a nonlinear viscoelastic foundation subjected to a time-dependent load $f(T)$ is shown in Figure 1. The foundation is taken as a nonlinear Winkler's foundation with linear-plus-cubic stiffness and viscous damping with three parameters as follows:

$$
P(X, T)=K_{1} W(X, T)+K_{3} W^{3}(X, T)+C \frac{\partial W(X, T)}{\partial T},
$$

where $P(X, T)$ is the foundation force induced per unit length of the beam, $T$ and $X$ are the time and spatial coordinates 
along the axis of the beam, respectively, $W(X, T)$ is the lateral displacement function of the beam, $K_{1}$ and $K_{3}$ are the linear and nonlinear parameters of the foundation, respectively, and $C$ is the damping coefficient of the foundation.

By using the Hamilton principle and employing the Euler-Bernoulli beam theory, one can obtain the differential equations of the motion as follows:

$$
\begin{aligned}
& E I \frac{\partial^{4} W}{\partial X^{4}}+\rho A \frac{\partial^{2} W}{\partial T^{2}}+K_{1} W+K_{3} W^{3}+C \frac{\partial W}{\partial T} \\
& \quad=f(T) \delta\left(X-X_{1}\right),
\end{aligned}
$$

where $E$ and $I$ are Young's modulus and the inertia of the beam, respectively, $\rho$ is the density of the beam, $A$ is the sectional area of the beam, $\delta\left(X-X_{1}\right)$ is the Dirac delta function used to address the location of the attenuation load, and $f(T)$ is the function of the time-dependent load. Eq. (2) can be applied to any form of load excitation acting at a fixed position.

For the convenience of further analysis, we introduce the dimensionless variables and parameters as follows:

$$
\begin{aligned}
x & =\frac{X}{L}, \\
w & =\frac{W}{L}, \\
t & =\frac{T}{L^{2} \sqrt{\rho A / E I}}, \\
k_{1} & =\frac{L^{4} K_{1}}{E I}, \\
\eta & =\frac{K_{3} L^{2}}{K_{1}}, \\
c & =\frac{C L^{2}}{\sqrt{\rho A E I}}, \\
f_{1}(t) & =\frac{f(T) L^{3}}{E I},
\end{aligned}
$$

where $x, t$, and $w$ are the dimensionless spatial coordinate, the dimensionless time, and the dimensionless lateral displacement function of the beam, respectively, $k_{1}$ is the dimensionless linear parameter of the foundation, $\eta$ is the ratio between the dimensionless nonlinear parameter of the foundation and the dimensionless linear parameter of the foundation, $c$ is the dimensionless damping coefficient of the foundation, and $f_{1}(t)$ is the dimensionless function of the time-dependent load. Eq. (2) can be transformed into the dimensionless equation as

$$
\begin{aligned}
& \frac{\partial^{4} w}{\partial x^{4}}+\frac{\partial^{2} w}{\partial t^{2}}+k_{1} w+\eta k_{1} w^{3}+c \frac{\partial w}{\partial t} \\
& =f_{1}(t) \delta\left(x-x_{1}\right) .
\end{aligned}
$$

The boundary conditions of the simply supported beam are

$$
\begin{aligned}
w(0, t) & =0, \\
\frac{\partial^{2} w(0, t)}{\partial x^{2}} & =0 \\
w(1, t) & =0, \\
\frac{\partial^{2} w(1, t)}{\partial x^{2}} & =0 .
\end{aligned}
$$

In these numerical examples, the initial conditions are

$$
\begin{gathered}
\left.w(x, t)\right|_{t=0}=0, \\
\left.w_{, t}(x, t)\right|_{t=0}=0 .
\end{gathered}
$$

\subsection{Statement of the Problem}

2.2.1. Galerkin Discretization. The Galerkin method is applied to (4) to discretize the system in a spatial coordinate and to obtain a system of ordinary differential equations in the time domain. The dimensionless beam lateral displacement is assumed in the following form:

$$
w(x, t)=\sum_{n=1}^{N} \phi_{n}(x) q_{n}(t),
$$

where $\phi_{n}(x)$ are the trial functions and $q_{n}(t)$ are the sets of generalized displacements of the beams. For simplification at the ends, $\phi_{n}(x)=\sin (n \pi x)$ [5] and $N$ is the Galerkin truncation term. In this research, the first $N$ terms are considered to determine $w(x, t)[4]$. Substituting (7) into (4) leads to

$$
\begin{gathered}
\sum_{n=1}^{N}\left\{\ddot{q}_{n}(t) \sin (n \pi x)+c \dot{q}_{n}(t) \sin (n \pi x)+\left[(n \pi)^{4}+k_{1}\right]\right. \\
\left.\cdot q_{n}(t) \sin (n \pi x)\right\}+\eta k_{1} \sum_{a=1}^{N} \sum_{b=1}^{N} \sum_{c=1}^{N} q_{a}(t) q_{b}(t) q_{c}(t) \\
\cdot \sin (a \pi x) \sin (b \pi x) \sin (c \pi x)=f_{1}(t) \delta\left(x-x_{1}\right) .
\end{gathered}
$$

Multiplying both sides of (8) by $\sin (m \pi x)$ ( $m$ is any positive integer less than $N$ ) and integrating $x$ from 0 to 1 , we obtain the following form:

$$
\begin{aligned}
& \frac{1}{2}\left\{\ddot{q}_{m}(t)+c \dot{q}_{m}(t)+\left[(m \pi)^{4}+k_{1}\right] q_{m}(t)\right\} \\
& \quad+\frac{1}{8} \eta k_{1} \sum_{a=1}^{N} \sum_{b=1}^{N} \sum_{c=1}^{N} q_{a}(t) q_{b}(t) q_{c}(t) \varphi(a, b, c, m) \\
& \quad=f_{1}(t) \sin \left(m \pi x_{1}\right), \\
& \varphi(a, b, c, m)=\phi(a+b-c-m)-\phi(a+b+c-m) \\
& \quad-\phi(a+b-c+m)-\phi(a-b+c+m) \\
& \quad-\phi(a-b-c-m)+\phi(a-b+c-m) \\
& \quad+\phi(a-b-c+m),
\end{aligned}
$$




$$
\phi(x)= \begin{cases}1, & x=0 \\ 0, & x \neq 0\end{cases}
$$

The initial conditions in (6) become

$$
q_{n}(0)=\dot{q}_{n}(0)=0 \quad \text { for } \mathrm{n}=1,2,3, \ldots, \mathrm{N}
$$

2.2.2. Runge-Kutta Method. The general system of fourthorder Runge-Kutta method can be written as

$$
\begin{aligned}
y_{n+1} & =y_{n}+h * \frac{\left(l_{1}+2 * l_{2}+2 * l_{3}+l_{4}\right)}{6}, \\
l_{1} & =f\left(t_{n}, y_{n}\right), \\
l_{2} & =f\left(t_{n}+\frac{h}{2}, y_{n}+l_{1} * \frac{h}{2}\right), \\
l_{3} & =f\left(t_{n}+\frac{h}{2}, y_{n}+l_{2} * \frac{h}{2}\right), \\
l_{4} & =f\left(t_{n}+h, y_{n}+\mathrm{h} * l_{3}\right),
\end{aligned}
$$

where $h$ is the time step of the calculation, $y_{n}$ is the value of the calculation of the time step in the $\mathrm{n}^{\text {th }}$ step, $y_{n+1}$ is the value of the calculation of the time step in the $(n+1)^{\text {th }}$ step, $l_{1}$ is the slope at the beginning of the period of time, $l_{2}$ is the slope at the midpoint of the period of time (the slope $l_{1}$ is used to determine the value of $y$ at point $t_{n}+h / 2$ by using Euler method), $l_{3}$ is also the slope at the midpoint of the period of time, but it uses slope $l_{2}$ to determine the value of $y$ at point $t_{n}+h / 2, l_{4}$ is the slope at the end of the period of time, and the value of $y$ is determined by $l_{3}$.

Eq. (9a) is now transformed into a unitary first-order system. Assuming $Q_{m}(t)=\dot{q}_{m}(t)$, then

$$
\begin{aligned}
& \dot{q}_{m}(t)=Q_{m}(t) \\
& \dot{Q}_{m}(t)=2 f(t) \sin \left(m \pi x_{1}\right)-c Q_{m}(t) \\
& -\left[(m \pi)^{4}+k_{1}\right] q_{m}(t) \\
& -\frac{1}{4} \eta k_{1} \sum_{a=1}^{\infty} \sum_{b=1}^{\infty} \sum_{c=1}^{\infty} q_{a}(t) q_{b}(t) q_{c}(t) \varphi(a, b, c, m) \text {. }
\end{aligned}
$$

Calculating the above equations by the fourth-order Runge-Kutta method, $Q_{m}(t)$ and $q_{m}(t)$ can be acquired. Then, the time series of the lateral displacement of each point on the beam are further obtained.

\section{Nonlinear Identification Method of a Euler-Bernoulli Beam Rested on a Nonlinear Viscoelastic Foundation Based on the Information Entropy}

3.1. Transfer Entropy. For two stationary Markov processes $x$ and $y$, when the historical information of the process $x$ is fully known, the transfer entropy $T_{y \rightarrow x}$ is used to quantify the additional description information for a certain state of the process $x$ in the future, which is provided by process $y$. According to the dynamic interdependence between processes $x$ and $y$, the formula of the transfer entropy [17] can be written as

$$
\begin{gathered}
T_{y \rightarrow x}\left(x(1) \mid x^{(k)}, y^{(l)}\right)=\iiint p\left(x(1), x^{(k)}, y^{(l)}\right) \\
\cdot \log _{2}\left(\frac{p\left(x(1) \mid x^{(k)}, y^{(l)}\right)}{p\left(x(1) \mid x^{(k)}\right)}\right) \mathrm{d} x(1) \mathrm{d} x^{(k)} \mathrm{d} y^{(l)},
\end{gathered}
$$

where $k$ represents the order of the Markov process $x$ and $l$ represents the order of the Markov process $y$. The studies on structural dynamic information by scholars such as Nichols $[13,16,18,19]$ and Overbey $[12,20]$ suggested that in order to avoid evaluating the high-dimensional probability density when calculating (13) and save calculation time, we could assume that both processes ( $x$ and $y$ ) are first-order Markov processes, namely, $k=l=1$. Under these assumptions, the transfer entropy of a single time factor can be obtained by adding a time delay $\tau$ to process $y$ in (13) at the same time as

$$
\begin{array}{r}
T_{y \rightarrow x}(x(1) \mid x, y(\tau))=\iiint p(x(1), x, y(\tau)) \\
\cdot \log _{2}\left(\frac{p(x(1) \mid x, y(\tau))}{p(x(1) \mid x)}\right) \mathrm{d} x(1) \mathrm{d} x \mathrm{~d} y(\tau),
\end{array}
$$

where $y(\tau)=y(n+\tau)$.

3.2. Kernel Density Estimation. The formula of transfer entropy, i.e., (14), does not require the response signal to be linear or consistent with the Gaussian distribution; it only needs to satisfy the stability. The calculation of the timedelayed transfer entropy involves an estimation of the multiple probability densities. Nichols [13] and Prichard et al. [21] proposed that the kernel density estimation method (a nonparametric density estimation method) can be used to solve the problem.

For each point in the data sequence $x(n)$, the kernel density can be estimated as follows:

$$
\begin{aligned}
& \widehat{p}(x(n), \varepsilon) \\
&=\frac{1}{N-2 h-1} \sum_{\substack{m=1 \\
|m-n|>\omega}}^{N} \Theta(\varepsilon-\|x(n)-x(m)\|),
\end{aligned}
$$

where $N$ is the length of the data sequence and $\Theta$ is the unit step function,

$$
\begin{aligned}
& \Theta(\varepsilon-\|x(n)-x(m)\|) \\
& \quad= \begin{cases}1: & \varepsilon-\|x(n)-x(m)\| \geq 0 \\
0: & \varepsilon-\|x(n)-x(m)\|<0 .\end{cases}
\end{aligned}
$$

The operator $\|\cdot\|$ uses the Euclidean norm approximation and the parameter $\omega$ represents a Theiler window, which is used to eliminate the deviation in the kernel density estimation 
[22]. The parameter $\varepsilon$ represents a fixed bandwidth. The larger the $\varepsilon$ is, the smoother the density function curve is with less variability. However, the deviation of the estimate is greater, so it is suggested that the range of the value of $\varepsilon$ is $2.5 \%-12.5 \%$ of the standard deviation of the time series [13]. Before the calculation, it is recommended that the mean and variance of the time series be converted to 0 and 1 , respectively, by preprocessing.

Prichard et al. [21] proposed that the Shannon entropy of the process $x(n)$ could be approximately expressed as

$$
\begin{gathered}
\int p(x(n)) \log _{2}(p(x(n))) \mathrm{d}(x(n)) \\
\approx \frac{1}{N} \sum_{n} \log _{2}(\hat{p}(x(n), \varepsilon)) .
\end{gathered}
$$

Substituting (17) into (14) and considering the conditional probability $p(a \mid b)=p(a, b) / p(b)$, the transfer entropy can be simplified to the function of the kernel density estimation:

$$
\begin{aligned}
& \hat{T E}(x(1) \mid x, y(T), \varepsilon)=\frac{1}{N} \\
& \quad \cdot \sum_{n}\left\{\log _{2}(\widehat{p}(x(n+1), x(n), y(n+T), \varepsilon))\right. \\
& \quad+\log _{2}(\widehat{p}(x(n), \varepsilon))-\log _{2}(\widehat{p}(x(n+1), x(n), \varepsilon)) \\
& \left.\quad-\log _{2}(\widehat{p}(x(n+1), y(n+T), \varepsilon))\right\} .
\end{aligned}
$$

Expanding (15), the probability density estimate of the first term on the right side of (18) can be written as

$$
\begin{aligned}
\log _{2}(\widehat{p}(x(n+1), x(n), y(n+T), \varepsilon)) \\
=\frac{1}{N} \sum_{\substack{m=1 \\
|m-n|>\omega}}^{N} \Theta\left(\varepsilon-\left\|\begin{array}{c}
x(n+1)-x(m+1) \\
x(n)-x(m) \\
y(n+T)-y(m+T)
\end{array}\right\|\right) .
\end{aligned}
$$

In general, the response data is limited; however, if such data can meet the stationarity and ergodicity assumptions, we can use the kernel density estimation method $[13,16]$. The method of kernel density estimation does not strictly require stationary and ergodicity data, so (18) can be used to study the nonlinear identification of rail foundation beams subjected to stationary excitation. Eq. (18) is applicable to both nonlinear data and linear data and can be regarded as a general algorithm.

3.3. Improved Surrogate Data Algorithm. For many practical structures, it is difficult to know the initial state of the monitoring data, so the damage identification methods based on the initial state of the structure can only be used for controlled nonlinear tests in the laboratory but not for the nonlinear analysis of the actual engineering structure. To solve this practical problem, Nichols et al. [13] used the kernel density estimation transfer entropy combined with an improved surrogate data algorithm [21-23] to conduct many effective research studies on nonlinear structural damage to remove the dependence of the initial state of the structure.
The improved surrogate data algorithm not only retains the linear correlation characteristic of the original signal but also retains the amplitude distribution characteristic of the original signal. This method is also applicable to general random data sequences without a Gaussian distribution, which is more applicable than the common surrogate data method. Referring to the research results of Schreiber et al. [24], we used the improved surrogate data algorithm to generate surrogate data for the computation.

3.4. Nonlinearity Index of the Transfer Entropy. Assuming that the transfer entropy computed from the improved surrogate data sets is consistent with the Gaussian distribution at each time delay, confidence intervals can be defined to quantitatively describe the nonlinearity of the structure. When the difference between the transfer entropy calculated from the surrogate data sets and the transfer entropy calculated from the original data set is located within the confidence interval at each time delay, the structure is consistent with a linear model. In contrast, the structure is assumed to be nonlinear at a desired level of confidence. We propose the initial nonlinearity index as

$$
\xi_{x_{i} \rightarrow x_{j}, n}(\tau)=\left|\frac{S_{x_{i} \longrightarrow x_{j}, n}(\tau)-D_{x_{i} \longrightarrow x_{j}}(\tau)}{\sigma_{0}(\tau)}\right|,
$$

where $\sigma_{0}(\tau)$ is the standard deviation of the transfer entropy values calculated from each set of 100 sets of the surrogate data of the baseline data set at time delay $\tau, S_{x_{i}-x_{j}, n}(\tau)$ is the transfer entropy calculated from the $\mathrm{n}^{\text {th }}$ set of the surrogate data sets at time delay $\tau$, and $D_{x_{i}-x_{j}}(\tau)$ is the transfer entropy calculated from the original data set at time delay $\tau$. For systems with unknown nonlinearity degree, the difference is calculated at the same time delay between the transfer entropy calculated from each set of the surrogate data sets and the transfer entropy calculated from the corresponding original data set. Eq. (20) shows the ratio of the absolute value of the difference to the standard deviation $\sigma_{0}(\tau)$ calculated from the baseline data. Eq. (20) can be used to measure the degree of dispersion of the transfer entropy values calculated from two kinds of data. In this paper, we used confidence intervals of $95 \%$. As a bilateral inspection problem, the critical point value $S=1.96 . \xi_{x_{i} \longrightarrow x_{j}, n}(\tau)$ is compared with this threshold, and the result provides a basis for judging whether the system has nonlinearity characteristics. To incorporate the confidence level, a nonlinearity index can be introduced such that nonzero values appear only above the threshold [12]:

$$
\begin{aligned}
Z_{x_{i}-x_{j}} & =\frac{1}{n_{0} \times\left(2 \tau_{0}+1\right) \times \alpha} \\
& \cdot \sum_{n=1}^{n_{0}} \sum_{\tau=-\tau_{0}}^{\tau_{0}}\left(\xi_{x_{i} \longrightarrow x_{j}, n}(\tau)-S\right) \Theta\left(\xi_{x_{i} \longrightarrow x_{j}, n}(\tau)-S\right),
\end{aligned}
$$

where $\Theta$ is the unit step function, $n_{0}$ is the number of the surrogate data sets, $\tau_{0}$ is the maximal time delay of the calculation, and $\alpha$ is the significance level. If $Z_{x_{i} \longrightarrow x_{j}}$ is greater 
TABLE 1

(a) Material properties of the rail

\begin{tabular}{lcc}
\hline Item & notation & value \\
\hline Young's modulus & $\mathrm{E}$ & $210 \mathrm{GPa}$ \\
Mass density & $\rho$ & $7850 \mathrm{~kg} / \mathrm{m}^{3}$ \\
Cross-sectional area & $A$ & $7.69 \times 10^{-3} \mathrm{~m}^{2}$ \\
Area moment of inertia & $I$ & $3.055 \times 10^{-5} \mathrm{~m}^{4}$ \\
Length & $L$ & $18 \mathrm{~m}$ \\
\hline
\end{tabular}

(b) Mechanical parameters of the foundation

\begin{tabular}{lccc}
\hline Item & notation & value & Dimensionless value \\
\hline Mean stiffness & $\mathrm{K}_{1}$ & $3.5 \times 10^{7} \mathrm{~N} / \mathrm{m}^{2}$ & $5.727 \times 10^{5}$ \\
Nonlinear stiffness & $\mathrm{K}_{3}$ & $4 \times 10^{14} \mathrm{~N} / \mathrm{m}^{4}$ & $2.1207 \times 10^{15}$ \\
Viscous damping & $\mathrm{C}$ & $1732 \times 10^{3} \mathrm{~N} \mathrm{~S} / \mathrm{m}^{2}$ & $2.8515 \times 10^{4}$ \\
\hline
\end{tabular}

TABLE 2: Nonlinearity degree and the corresponding ratio between the dimensionless nonlinear parameter and the dimensionless linear parameter of the foundation.

\begin{tabular}{lcccccccc}
\hline Nonlinearity degree & 0 & 1 & 2 & 3 & 4 & 5 & 6 \\
\hline Ratio $(\eta)$ & 0 & $10^{8}$ & $5 \times 10^{8}$ & $10^{9}$ & $2 \times 10^{9}$ & $3 \times 10^{9}$ & $3.7029 \times 10^{9}$ & $5 \times 10^{9}$ \\
\hline
\end{tabular}

than 0 , the transmission relationship between the two sets of signals has a nonlinear characteristic; therefore, the structure has a nonlinear characteristic at the 95 percent confidence level. If the baseline data has nonlinearity characteristics, $Z_{x_{i} \longrightarrow x_{j}}$ can be used to determine whether the nonlinearity degree of system increases.

\section{Numerical Results and Discussion}

\subsection{Influence of the Nonlinear Parameter of the Foundation on the Transfer Entropy}

4.1.1. Calculation Parameters of the Model. The beam is assumed to be the UIC60 European high-speed rail. The material properties of the rail are shown in Table 1(a), and the mechanical parameters of the foundation are shown in Table 1(b). Because the differential equation of the system motion is dimensionless, a cosine excitation with time-dependent frequency which is expressed as $f(t)=$ $60 \cos \{[(70-50) t / 3+50] t \times 2 \pi\}$ is applied at the mid-span of the beam. The calculation time is 3 , and 4000 samples are taken per unit time. Because this model is a symmetric structure, the results of the time response of the lateral displacement at points $1 / 10,2 / 10,3 / 10$, and $4 / 10$ of the beam (numbered 1, 2, 3, and 4, respectively, from left to right, and see Figure 1) are selected, and then the time-delayed transfer entropy is calculated from the time-history results. To simulate different nonlinearity levels of the system, the ratios $\eta$ between the dimensionless nonlinear parameter and the dimensionless linear parameter of the foundation are taken as $0,10^{8}, 5 \times 10^{8}, 10^{9}, 2 \times 10^{9}, 3 \times 10^{9}, 3.7029 \times$ $10^{9}, 5 \times 10^{9}$, and the corresponding nonlinearity degrees of the system are $0,1,2,3,4,5,6$ and 7 , respectively (see Table 2 ). The value $3.7029 \times 10^{9}$ is the ratio $\eta$ between the dimensionless

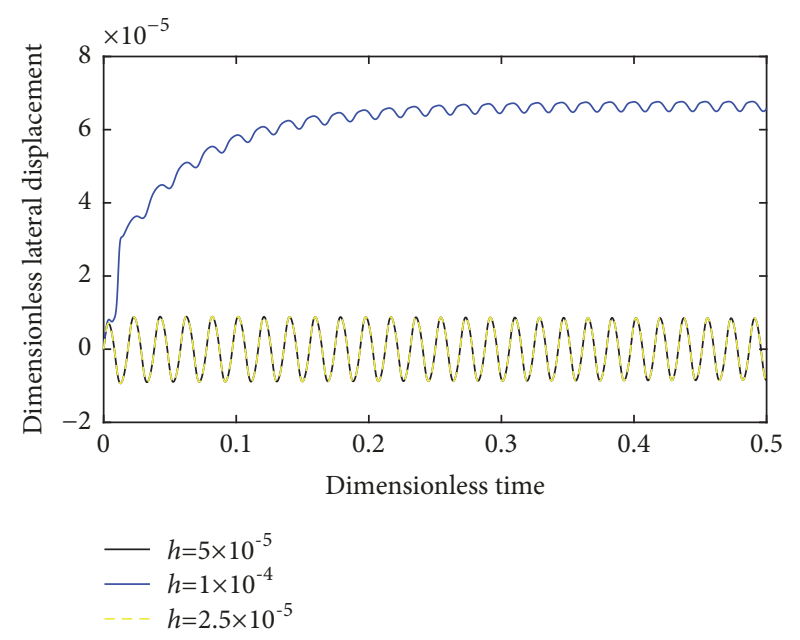

FIGURE 2: Effect of the time step on the dimensionless lateral displacements of sampling point 3 .

nonlinear parameter and the dimensionless linear parameter of the foundation shown in Table 1(b).

The emphasis of this paper is to use the time-delayed transfer entropy combined with the surrogate data algorithm to identify the nonlinearity degree of the beam on a nonlinear foundation. To meet a certain accuracy requirement, the time response results of the 11-term Galerkin method are sufficiently accurate to be used in the transfer entropy calculation. Figure 2 shows the effects of the time step of the calculation using fourth-order Runge-Kutta method on the lateral displacements of sampling point 3 , and other calculation parameters use the parameter values shown in Table 1. It shows that the calculation does not converge when the time step $h$ is taken as $1 \times 10^{-4}$, and the calculation curves of 

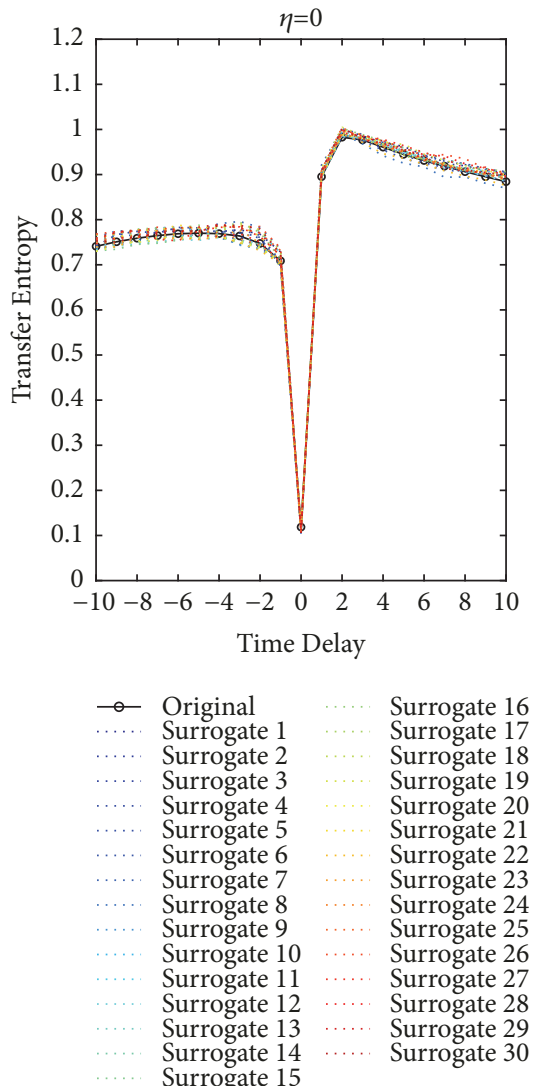

(a) Nonlinearity degree 0

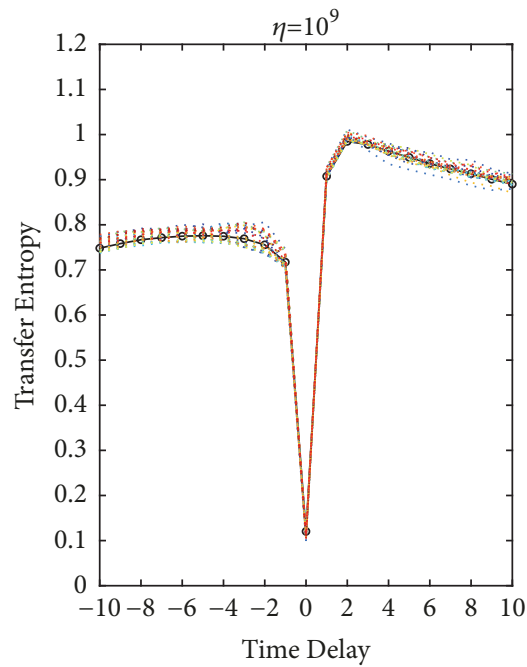

Original Surrogate 1 Surrogate 2 Surrogate 3 Surrogate 4 Surrogate 5

Surrogate 6

Surrogate 7 Surrogate 8 Surrogate 9 Surrogate 10 Surrogate 11 Surrogate 12 Surrogate 13 Surrogate 14 Surrogate 15 Surrogate 17 Surrogate 18 Surrogate 19 Surrogate 20 Surrogate 21 Surrogate 22 Surrogate 23 Surrogate 24 Surrogate 25 Surrogate 26 Surrogate 27 Surrogate 28 Surrogate 29 Surrogate 30

(d) Nonlinearity degree 3
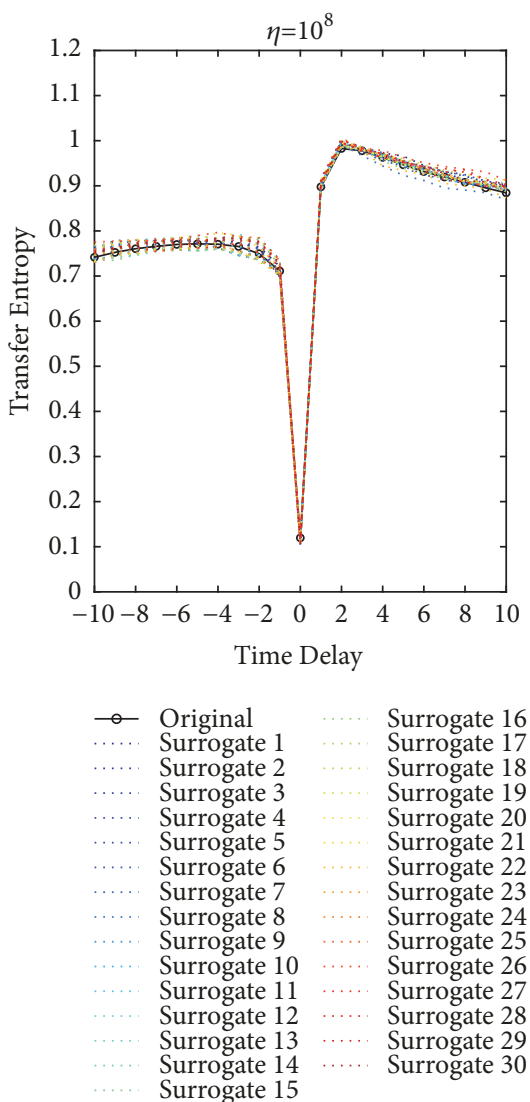

(b) Nonlinearity degree 1

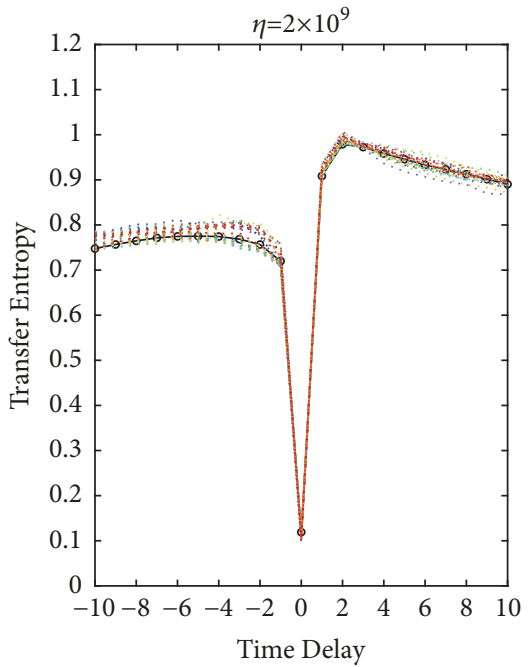

Original Surrogate 1 Surrogate 2

Surrogate 3

Surrogate 4

Surrogate 5

Surrogate 6

Surrogate 7

Surrogate 8

Surrogate 9

Surrogate 10

Surrogate 11

Surrogate 12

Surrogate 13

Surrogate 14

(e) Nonlinearity degree 4

Surrogate 16 Surrogate 17 Surrogate 18 Surrogate 19 Surrogate 20 Surrogate 21

Surrogate 22

Surrogate 23 Surrogate 24 Surrogate 25 Surrogate 26 Surrogate 27 Surrogate 28 Surrogate 29 Surrogate 30

Fugure 3: Continued.
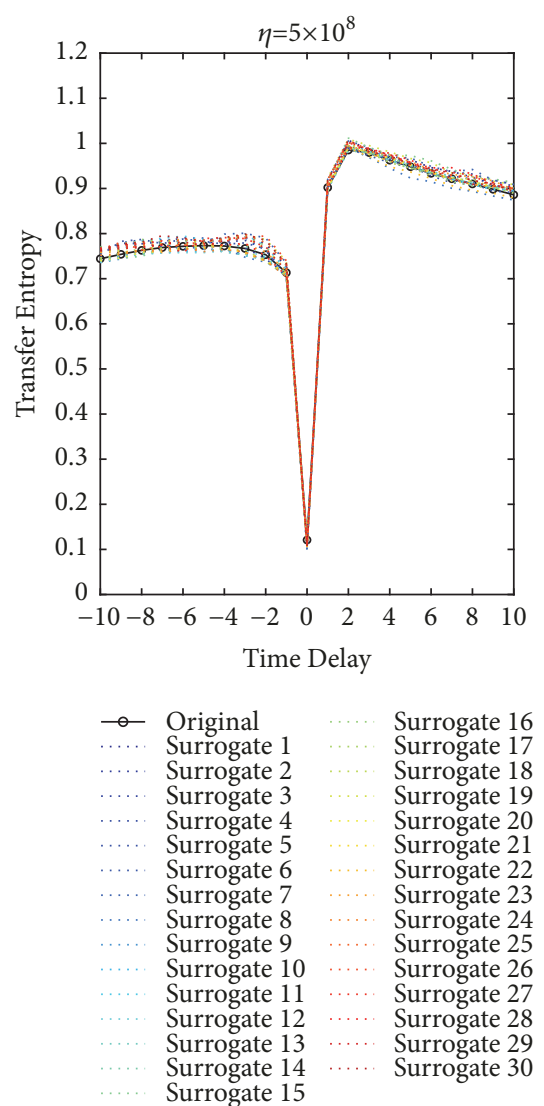

(c) Nonlinearity degree 2

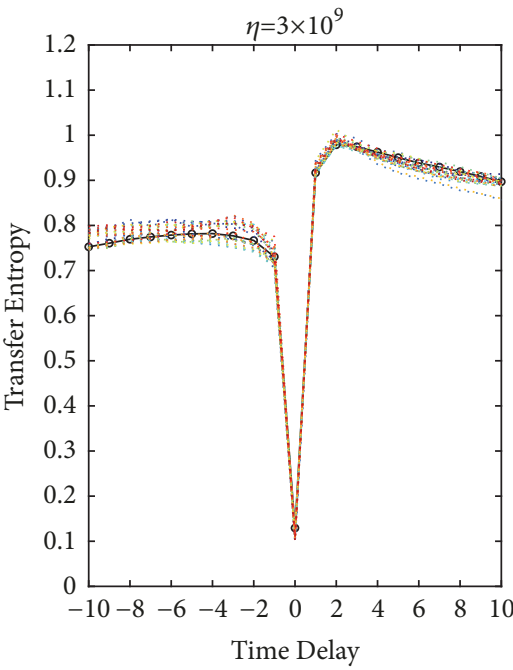

Original Surrogate $4 \ldots .$. Surrogate 21 Surrogate 22 $\begin{array}{lll}\text { Surrogate } 7 & \ldots . . . & \text { Surrogate } 23\end{array}$ Surrogate 8 …. Surrogate 24 Surrogate $9 \quad \cdots .$. Surrogate 25 Surrogate 10 …. Surrogate 26 Surrogate $11 \quad \ldots .$. Surrogate 27 Surrogate 12 …... Surrogate 28 Surrogate 13 …... Surrogate 29 Surrogate $14 \quad$ Surrogate 30 $\begin{array}{lll}\text { Surrogate } 14 & \cdots \cdots . . . & \text { Surrogate } 30 \\ \text { Surrogate } 15 & & \end{array}$

(f) Nonlinearity degree 5 


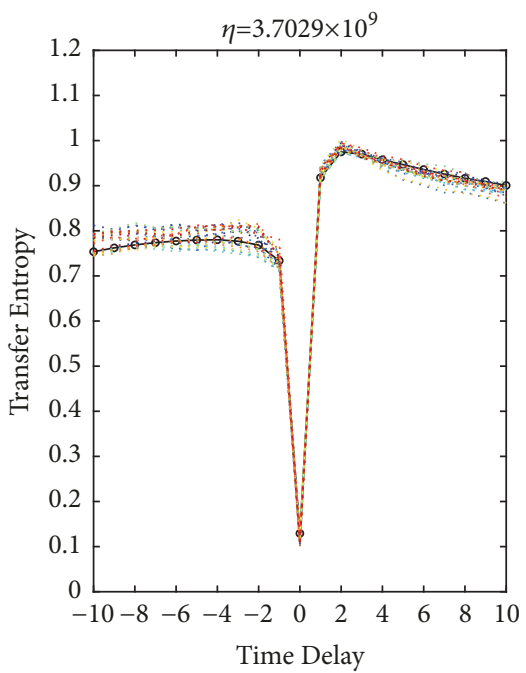

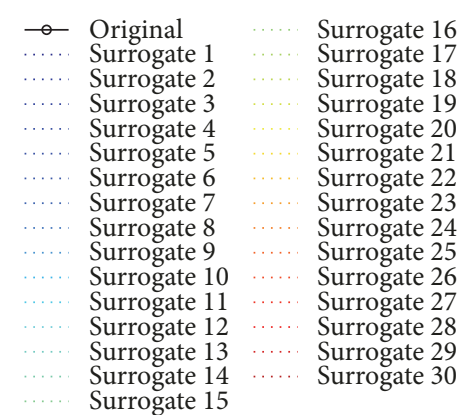

(g) Nonlinearity degree 6
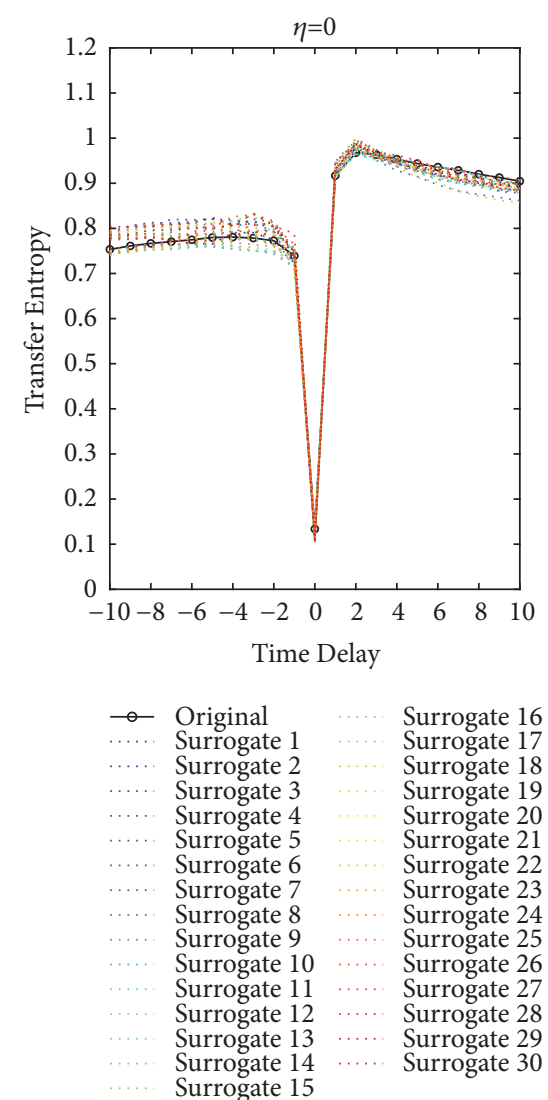

(h) Nonlinearity degree 7

FIGURE 3: The transfer entropy $T_{x_{3} \rightarrow x_{1}}$ between sampling point 3 and sampling point 1 at different nonlinearity degrees.

the other two cases are approximately coincident. Therefore, the subsequent calculations in this paper are calculated using the time step $h=5 \times 10^{-5}$. The smaller the time step, the higher the sampling frequency. In the same calculation time delay scale, the transfer entropy calculated from the time response data collected at the low sampling frequency will have a local minimum at more time delays. However, the location of the local minimum is often a sensitive area for judging whether system has nonlinearity characteristics. When the nonlinearity level of the system is low, compared with the time response data collected at the higher sampling frequency, the time response data collected at the lower sampling frequency are more conducive to the nonlinearity identification function of the transfer entropy method. When the nonlinearity level of the system is high, the time response data collected at the lower sampling frequency lose too much detailed information. The nonlinearity level of the system in this example is unknown, so the time step should not be too small. Otherwise, the nonlinearity identification function of the transfer entropy method may not be effective. In this paper, the length of the lateral displacement time response data is chosen to be 8000 for the calculation of the transfer entropy. The time response data calculated using the time step $h=5 \times 10^{-5}$ is taken as a point every 5 points, which is equivalent to 4,000 samples per unit time.
The ADF (Augmented Dickey-Fuller) test shows that both the original data set and the surrogate data sets of the lateral displacement time response series of the model are in accordance with the requirement of stationarity (the confidence level is 99\%), which is suitable for the calculation of the kernel density estimation transfer entropy combined with the improved surrogate data algorithm for nonlinear identification analysis. When calculating with the kernel density estimation method, the size of the Theiler window $\omega$ is taken as 90 , and the fixed band width $\varepsilon$ is taken as 0.09 .

4.1.2. Calculation Results of the Transfer Entropy. The transfer entropies computed from the original data and the surrogate data were carried out between any two sets of the timehistory response results of the four sampling points shown in Figure 1. Figure 3 shows the time-delayed transfer entropy $T_{x_{3} \longrightarrow x_{1}}$ between sampling point 3 and sampling point 1 at different nonlinearity levels. The horizontal axis of each subgraph represents the time delay, and the vertical axis represents the value of the transfer entropy at different time delays. Since (2) considers the damping factor, when the ratio $\eta$ between the dimensionless nonlinear parameter of the foundation and the dimensionless linear parameter of the foundation is zero, i.e., the nonlinearity degree of the system is 0 , the system is not completely linear, resulting in the 
TABLE 3: The ratio between the average of the absolute values of the nonlinear restoring force term $\left(\eta k_{1} w^{3}\right)$ and the average of the absolute values of the linear restoring force term $\left(k_{1} w\right)$ at the position of sampling point 4 at different nonlinearity degrees.

\begin{tabular}{lcccccccc}
\hline Nonlinearity degree & 0 & 1 & 2 & 3 & 4 & 5 & 6 & 7 \\
\hline The ratio $\lambda$ & 0 & 0.0048 & 0.0238 & 0.0472 & 0.0923 & 0.1349 & 0.1632 & 0.2123 \\
\hline
\end{tabular}

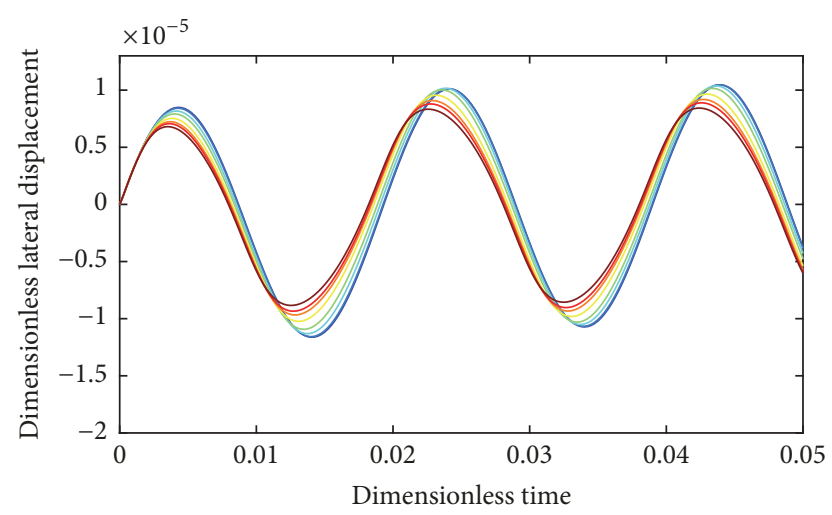

$\begin{array}{cr}\text { Nonlinear degree } 0 & \text { Nonlinear degree } 4 \\ \text { Nonlinear degree 1 } & \text { Nonlinear degree } 5 \\ \text { Nonlinear degree 2 } & \text { Nonlinear degree 6 } \\ \text { Nonlinear degree 3 } & - \text { Nonlinear degree 7 }\end{array}$

FIgURE 4: Dimensionless lateral displacement response of sampling point 3 at different nonlinearity degrees.

case that the time-delayed transfer entropy calculated from the original data and the surrogate data is not completely coincident. Here, the nonlinearity degree 0 is regarded as the baseline data for nonlinearity degree identification. Figure 4 shows the dimensionless lateral displacement response of sampling point 3 for a dimensionless calculation time of 0.05 . The horizontal axis represents the dimensionless calculation time, and the vertical axis represents the result of the dimensionless lateral displacement time-history response. Figure 4 shows that the time response results of nonlinearity degrees 0 and 1 are approximately equal. Because the ratio $\eta$ at nonlinearity degree 1 is only $2.7 \%$ of the ratio $\eta$ at nonlinearity degree 6 , the nonlinear force component caused by the nonlinear parameter of the foundation is relatively small compared to the nonlinear force component caused by the damping, leading to very similar results of the transfer entropy $T_{x_{3} \rightarrow x_{1}}$ at nonlinearity degrees 0 and 1 in Figure 3. With an increase in the ratio $\eta$, the nonlinear force caused by the nonlinear parameter of the foundation gradually increases, which leads to an increasingly obvious nonlinear characteristic in the time-varying response. Therefore, the differences in the results of the transfer entropy calculated from the original data and the surrogate data have become increasingly larger. During the process that the ratio $\eta$ increases from $5 \times 10^{8}$ to $5 \times 10^{9}$, the differences in the values of the transfer entropy calculated from the 30 sets of surrogate data are generally larger at each time delay, and the differences between the transfer entropy calculated from the surrogate data and the transfer entropy calculated from the original data also become larger.
The nonlinearity index constructed by (20) and (21) is plotted in Figure 5. The subgraphs show the final nonlinearity index $Z_{x_{i} \longrightarrow x_{j}}$ calculated from the transfer entropy $T_{x_{i} \longrightarrow x_{j}}$. The horizontal axis of each subgraph represents sampling point $i$, and the vertical axis represents sampling point $j$. The values of the transfer entropy calculated from the lateral displacement time response result measured at the same sampling point are always 0 . According to the definition of the transfer entropy, the signal itself does not transfer information. The values of the nonlinearity index calculated from the transfer entropy between different sampling points are all greater than 0 , indicating that the system already has nonlinearity. When the ratio $\eta$ is 0 , the nonlinearity index values are greater than 0 due to the existence of viscous damping of the foundation. Figure 6 shows the nonlinearity indexes between different sampling points corresponding to the nonlinearity degrees 0 to 7. The horizontal axis represents the sampling point group, and $i \longrightarrow j$ means that information is transferred from sampling point $i$ to sampling point $j$. The vertical axis represents the nonlinearity index value. The increasing trend of the nonlinearity index values of each sampling point group is very pronounced as the nonlinearity degree increases from degree 2. Because each set of the surrogate data is randomly generated, the quality of the data, to some degree, is generated by chance. Only when the number of data sets generated is large, the statistical law of the data can become stable. Thirty sets of surrogate data are generated in this study, although there is no guarantee that the contingency of the transfer entropy calculated from them is completely excluded.

The ratios $\lambda$ between the average of the absolute values of the nonlinear restoring force term $\left(\eta k_{1} w^{3}\right)$ of the foundation and the average of the absolute values of the linear restoring force term $\left(k_{1} w\right)$ of the foundation at the position of sampling point 4 of (4) at different nonlinearity degrees are shown in Table 3. Figure 7 is obtained from accumulating the nonlinearity index values of each sampling point group, and the horizontal axis is the ratio $\lambda$. Figure 7 shows that the sum of the nonlinearity index values increases as the ratio increases. This approach excludes the contingency of the surrogate data algorithm for most degrees, and the results also prove that the method based on the transfer entropy and surrogate data algorithm exhibits good stability.

\subsection{Influence of Young's Modulus of the Foundation on the Transfer Entropy}

4.2.1. Calculation Parameters of the Model. To analyze the influence of Young's modulus of the beam on the transfer entropy calculation, seven different Young's modulus values of the beam were collected, as shown in Table 4, and the corresponding case numbers are also shown in Table 4 . The 


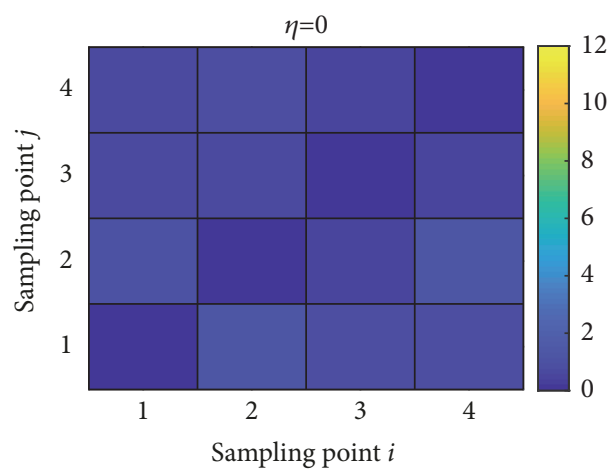

(a) Nonlinearity degree 0

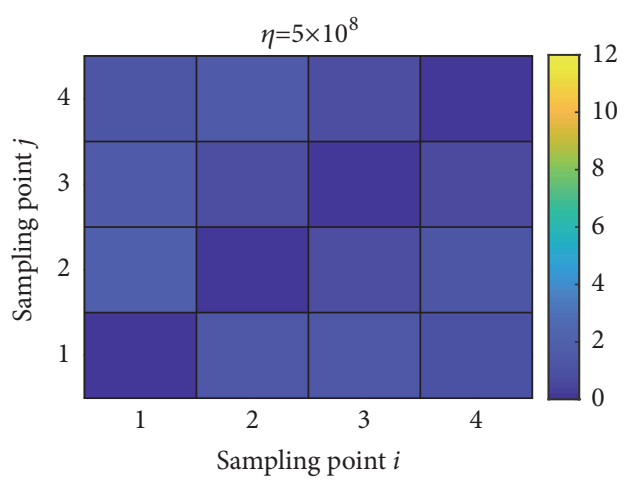

(c) Nonlinearity degree 2

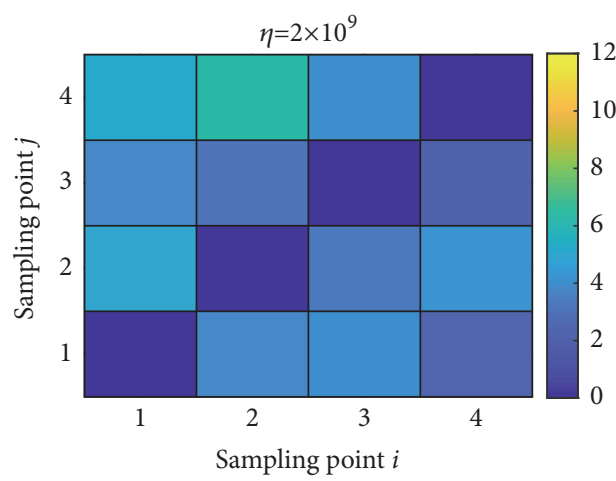

(e) Nonlinearity degree 4

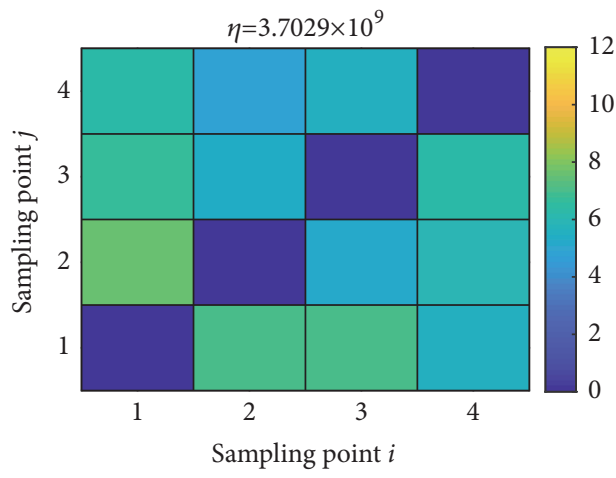

(g) Nonlinearity degree 6

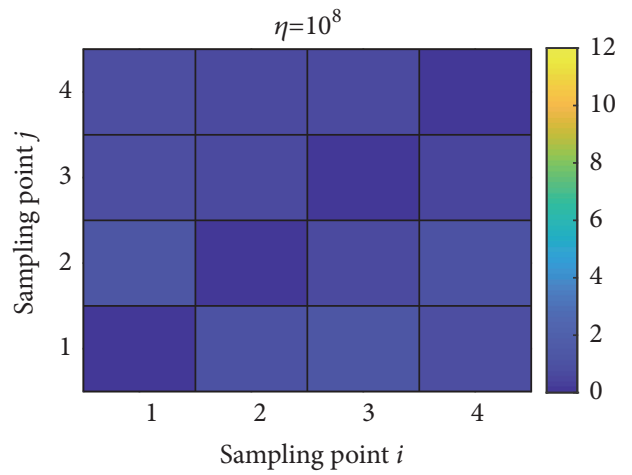

(b) Nonlinearity degree 1

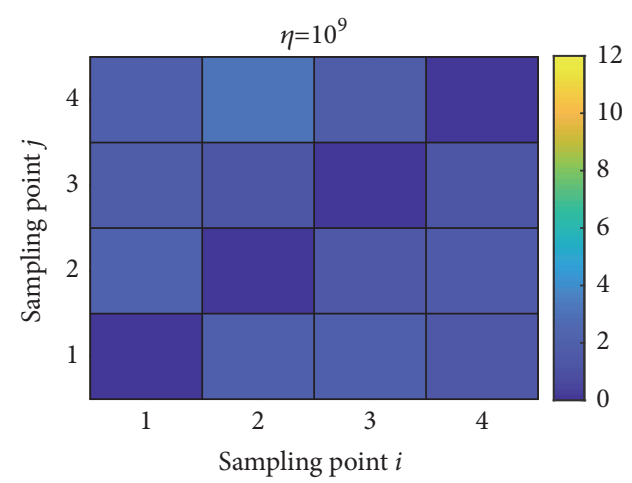

(d) Nonlinearity degree 3

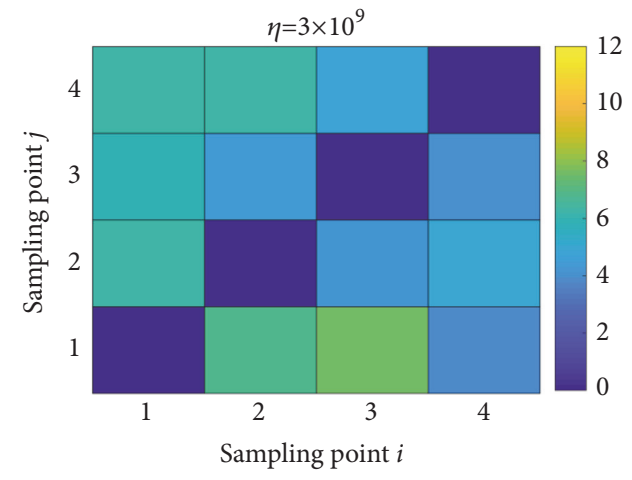

(f) Nonlinearity degree 5

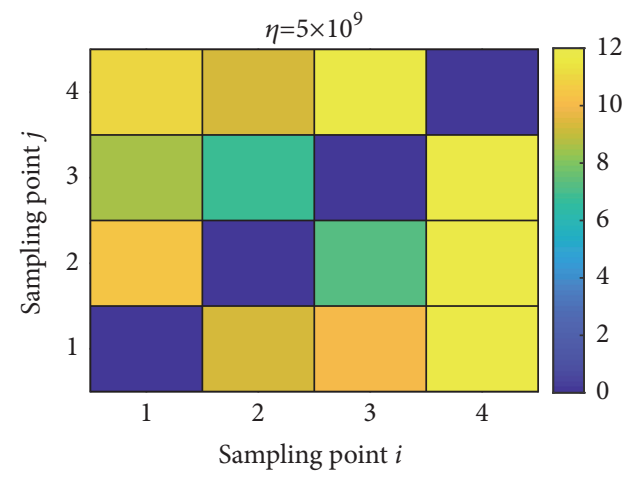

(h) Nonlinearity degree 7

FIGURE 5: Nonlinearity index of different nonlinearity degrees. 
TABLE 4: Values of Young's modulus.

\begin{tabular}{lccccccc}
\hline Case & 1 & 2 & 3 & 4 & 5 & 6 & 7 \\
\hline Young's modulus $(\mathrm{GPa})$ & 150 & 170 & 190 & 210 & 230 & 250 & 270 \\
\hline
\end{tabular}

TABLE 5: Properties of the rail and foundation.

\begin{tabular}{lcc}
\hline Item & notation & value \\
\hline Mass density & $\rho$ & $7850 \mathrm{~kg} / \mathrm{m}^{3}$ \\
Cross-sectional area & $A$ & $7.69 \times 10^{-3} \mathrm{~m}^{2}$ \\
Area moment of inertia & $I$ & $3.055 \times 10^{-5} \mathrm{~m}^{4}$ \\
Length & $L$ & $18 \mathrm{~m}$ \\
Mean stiffness & $K_{1}$ & $3.5 \times 10^{7} \mathrm{~N} / \mathrm{m}^{2}$ \\
Nonlinear stiffness & $K_{3}$ & $4 \times 10^{14} \mathrm{~N} / \mathrm{m}^{4}$ \\
Viscous damping & $C$ & $1732 \times 10^{3} \mathrm{~N} \mathrm{~S} / \mathrm{m}^{2}$ \\
\hline
\end{tabular}

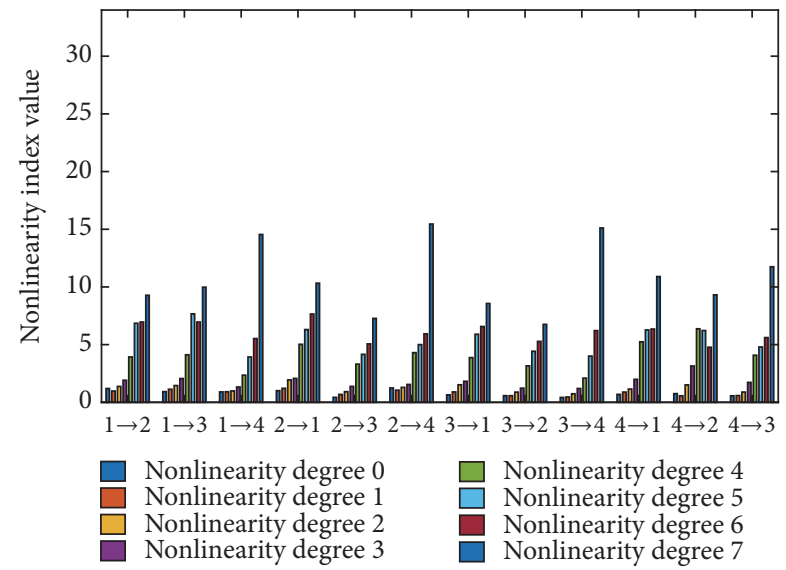

FIGURE 6: Comparison of the nonlinearity index values of different nonlinearity degrees.

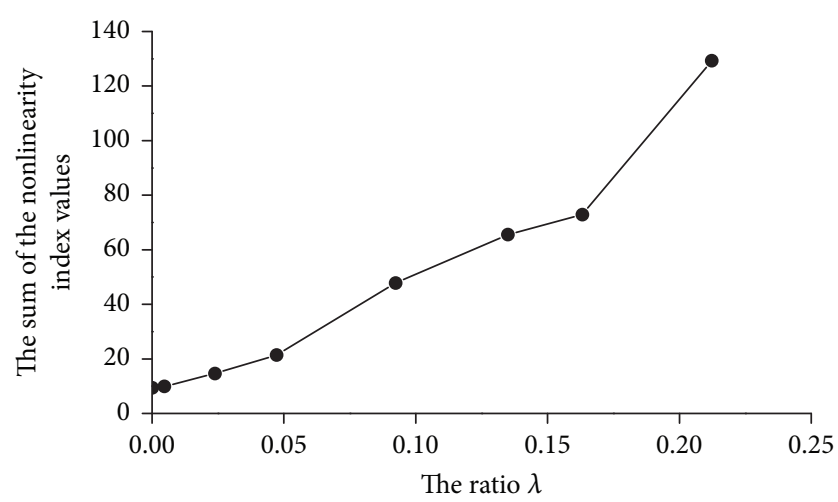

FIGURE 7: Sum of the nonlinearity index values of each sampling point group as a function of the ratio $\lambda$.

remaining calculation parameters are shown in Table 5. The load, calculation time, and sampling frequency are the same as in the above calculation model. The ADF (Augmented Dickey-Fuller) test shows that both the original data and the surrogate data of the lateral displacement time response series calculated with different Young's moduli are in accordance

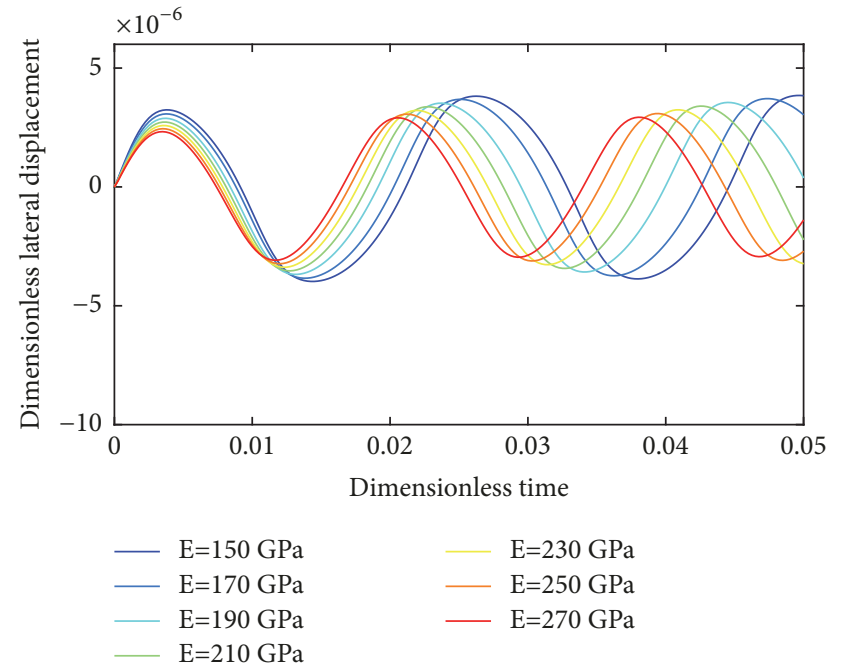

FIGURE 8: Effects of Young's modulus on the dimensionless lateral displacement response of sampling point 3 .

with the requirement of stationarity (the confidence level is $99 \%)$.

4.2.2. Calculation Results of the Transfer Entropy. Figure 8 shows the dimensionless lateral displacement response for different Young's moduli at the position of sampling point 3 (see Figure 1) for a dimensionless calculation time of 0.05 . In (2), except for Young's modulus, the other parameters remain unchanged. As Young's modulus of the beam increases, the amplitude of the dimensionless lateral displacement of the point on the beam gradually decreases. For the calculation results of different Young's moduli, the transfer entropies computed from the original data and the surrogate data are carried out between any two sets of the time-history response of the four sampling points shown in Figure 1. The nonlinearity degree 0 of the above example is taken as the baseline data, and the results of nonlinearity index are plotted as Figure 9.

Figure 10 shows the values of the nonlinearity index between the different sampling points of the different Young's 


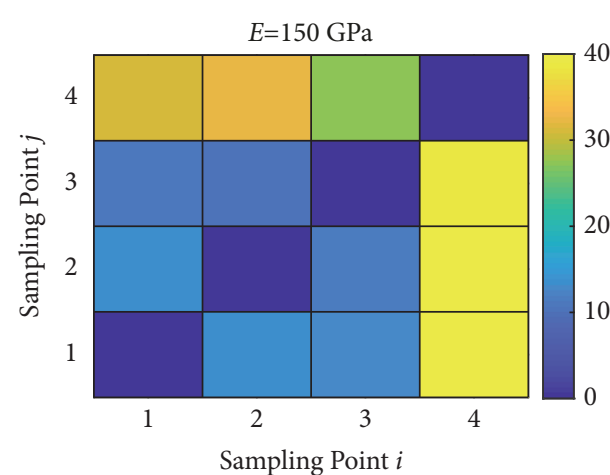

(a) Case 1

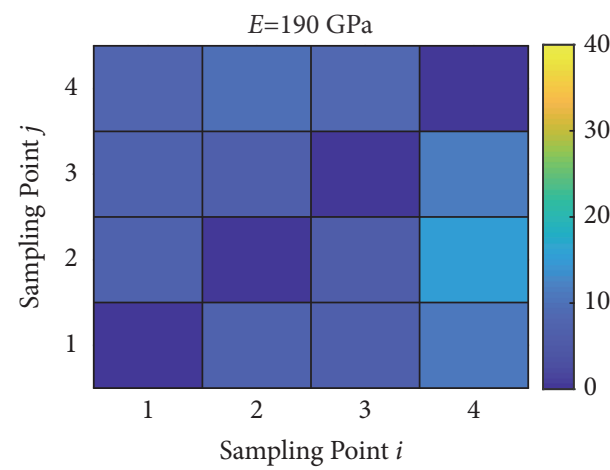

(c) Case 3

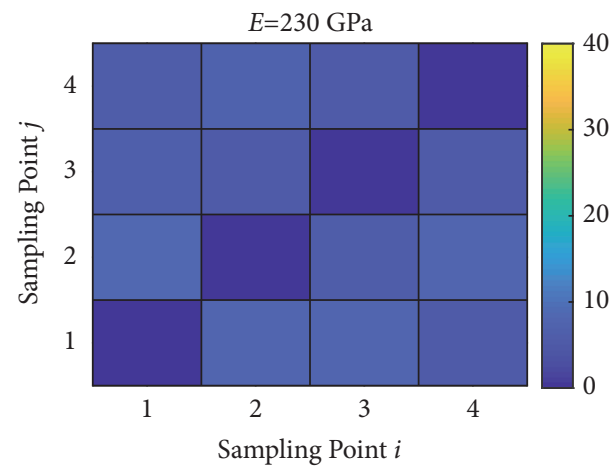

(e) Case 5

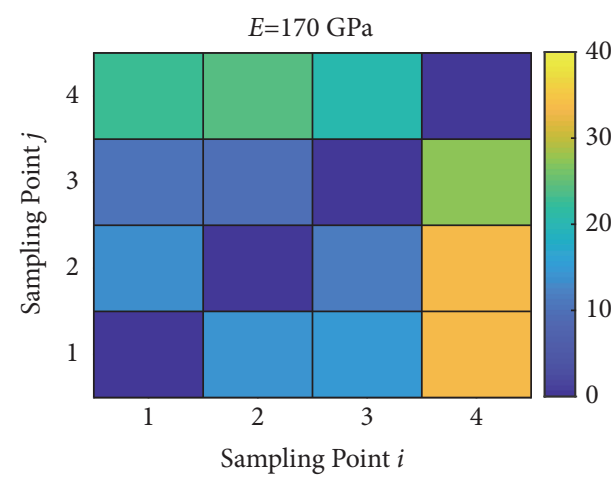

(b) Case 2

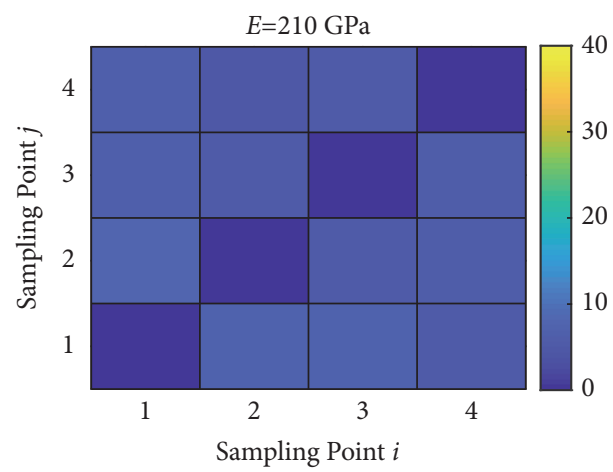

(d) Case 4

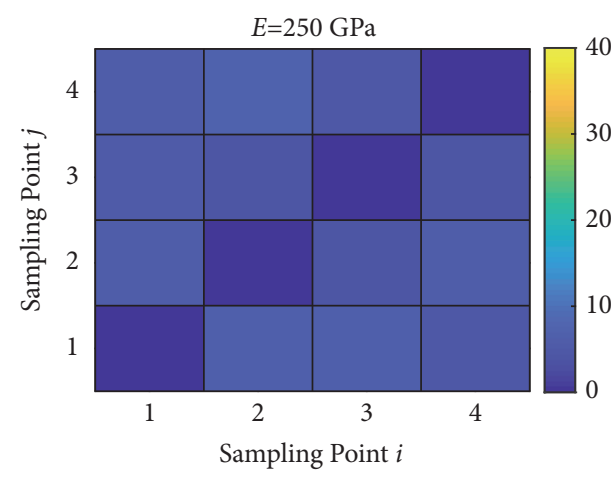

(f) Case 6

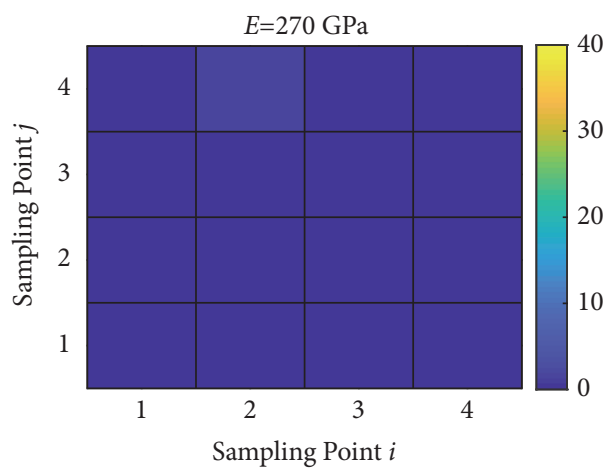

(g) Case 7

FIGURE 9: Nonlinearity index of each sampling point group in different cases. 


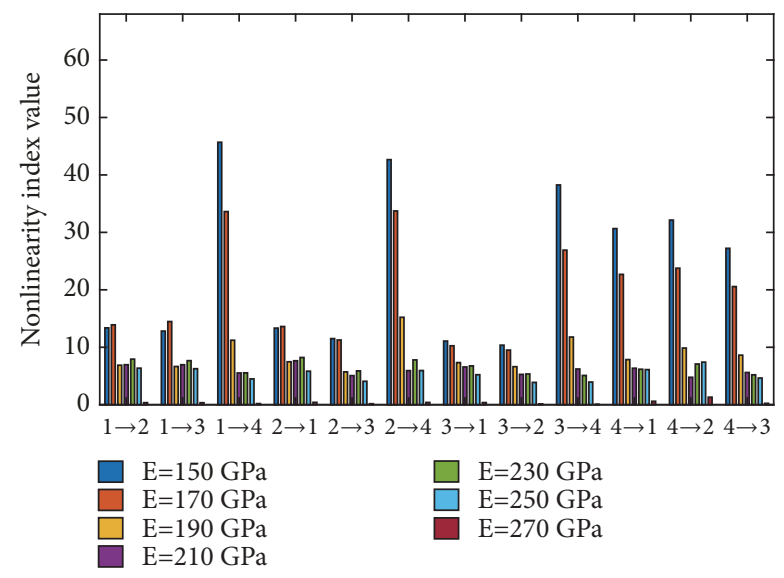

FIGURE 10: Comparison of the nonlinearity index values in different cases.

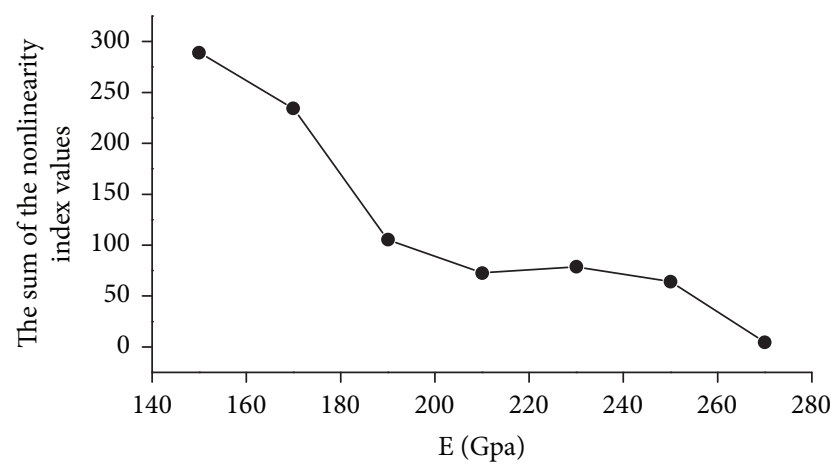

FIGURE 11: Sum of the nonlinearity index values of each sampling point group as a function of Young's modulus.

moduli. The horizontal axis represents the sampling point group, and the vertical axis represents the nonlinearity index value. In cases 4,5 , and 6 , the nonlinearity index values of each sampling point group change slightly with no obvious trend in the change. In case 3, compared to the other three cases mentioned above, the nonlinearity index values of some sampling point groups increase significantly. In case 2, compared to the other four cases mentioned above, the nonlinearity index values of all sampling point groups increase significantly. In case 1, compared to the other cases, the nonlinearity index values of most sampling point groups reach a maximum value. In case 7 , the nonlinearity index values of all sampling point groups reach the minimum value.

Figure 11 is obtained from accumulating the nonlinearity index values of each sampling point group in each case. As the value of Young's modulus becomes larger, the sum of the nonlinearity index values of each sampling point group generally decreases, indicating that the nonlinearity level of the system also decreases. The nonlinearity level of the system is mainly determined by the fourth and fifth terms on the left of (2). The value of the fourth term has a cubic relation with the lateral displacement value. Since the amplitude of lateral displacement at each point on the beam decreases with an increase in the value of Young's modulus, the nonlinear characteristic caused by the fourth term on the left side of (2) approximately decreases with the third power. The results demonstrate that the fourth term on the left of (2) may play a critical role in the nonlinearity level of the system when Young's modulus increases from $150 \mathrm{GPa}$ to $270 \mathrm{GPa}$.

\section{Conclusion}

According to the above results, it can be demonstrated that the Galerkin method combined with the fourth-order RungeKutta method is efficient in calculating the dynamic response of a homogeneous Euler-Bernoulli simply supported beam that is rested on a nonlinear viscoelastic foundation and subjected to a time-dependent load. In this paper, a different ratio between the dimensionless nonlinear parameter of the foundation and the dimensionless linear parameter of the foundation is used first to simulate the different nonlinearity levels of the system. Then, the nonlinearity level of the system is identified by a method based on the time-delayed transfer entropy and improved surrogate data algorithm, computing the transfer entropy on both the original data set and the surrogate data sets of the dynamic response results under different nonlinearity degrees of the system. Based on the difference between the transfer entropy results computed on both the original data set and the surrogate data sets, we present a nonlinearity index and use this index to analyze the transfer entropy results. It can be found that the nonlinearity index value increases with an increase of the nonlinearity degree of the system. By comparing the results of the transfer entropy of beams with different Young's modulus values, we also find that the method based on the time-delayed transfer entropy and surrogate data algorithm can effectively identify the nonlinearity degree of the system caused by the change in the mechanical properties of the beams. The improved surrogate data algorithm used in this paper can remove the dependence on the initial state of the structure and increase the applicability of the method based on the time-delayed transfer entropy and surrogate data in the field of nonlinear identification.

\section{Data Availability}

The data used to support the findings of this study are available from the corresponding author upon request.

\section{Conflicts of Interest}

The authors declare that there are no conflicts of interest regarding the publication of this article.

\section{Acknowledgments}

The authors gratefully acknowledge the financial support provided by the 973 Program of China (No. 2013CB035901), the Fundamental Research Funds for the Central Universities (No. 51679214), and the National Natural Science Foundation of China (No. 51379185). 


\section{References}

[1] L.-A. Wong and J.-C. Chen, "Damage identification of nonlinear structural systems," AIAA Journal, vol. 38, no. 8, pp. 1444-1452, 2000.

[2] M. H. Kargarnovin, D. Younesian, D. J. Thompson, and C. J. C. Jones, "Response of beams on nonlinear viscoelastic foundations to harmonic moving loads," Computers \& Structures, vol. 83, no. 23, pp. 1865-1877, 2005.

[3] A. D. Senalp, A. Arikoglu, I. Ozkol, and V. Z. Dogan, "Dynamic response of a finite length euler-bernoulli beam on linear and nonlinear viscoelastic foundations to a concentrated moving force," Journal of Mechanical Science and Technology, vol. 24, no. 10, pp. 1957-1961, 2010.

[4] M. Ansari, E. Esmailzadeh, and D. Younesian, "Internalexternal resonance of beams on non-linear viscoelastic foundation traversed by moving load," Nonlinear Dynamics, vol. 61, no. 1, pp. 163-182, 2010.

[5] S. M. Abdelghany, K. M. Ewis, A. A. Mahmoud, and M. M. Nassar, "Dynamic response of non-uniform beam subjected to moving load and resting on non-linear viscoelastic foundation," Beni-Suef University Journal of Basic and Applied Sciences, vol. 4, no. 7, pp. 192-199, 2015.

[6] S. F. Masri and T. K. Caughey, "A nonparametric identification technique for nonlinear dynamic problems," Journal of Applied Mechanics, vol. 46, no. 2, pp. 433-447, 1979.

[7] S. F. Masri, H. Sassi, and T. K. Caughey, "Nonparametric identification of nearly arbitrary nonlinear systems," Journal of Applied Mechanics, vol. 49, no. 3, pp. 619-628, 1982.

[8] S. F. Masri, R. K. Miller, A. F. Saud, and T. K. Caughey, "Identification of Nonlinear Vibrating Structures: Part I-Formulation," Journal of Applied Mechanics, vol. 109, no. 4, pp. 918-922, 1987.

[9] S. F. Masri, R. K. Miller, A. F. Saud, and T. K. Caughey, "Identification of nonlinear vibrating structures: Part II-applications," Journal of Applied Mechanics, vol. 54, no. 4, pp. 923-929, 1987.

[10] K. S. Mohammad, K. Worden, and G. R. Tomlinson, "Direct parameter estimation for linear and non-linear structures," Journal of Sound and Vibration, vol. 152, no. 3, pp. 471-499, 1992.

[11] N. E. Huang, Z. Shen, S. R. Long et al., "The empirical mode decomposition and the Hilbert spectrum for nonlinear and non-stationary time series analysis," Proceedings: Mathematical, Physical and Engineering Sciences, vol. 454, no. 1971, pp. 903995, 1998.

[12] L. A. Overbey and M. D. Todd, "Dynamic system change detection using a modification of the transfer entropy," Journal of Sound and Vibration, vol. 322, no. 1-2, pp. 438-453, 2009.

[13] J. M. Nichols, M. Seaver, and S. T. Trickey, "A method for detecting damage-induced nonlinearities in structures using information theory," Journal of sound \& Vibration, vol. 297, no. 1, pp. 1-16, 2006.

[14] Z.-K. Xie, G.-H. Liu, and Z.-G. Wu, "Dynamic damage identification for beam structures based on transfer entropy," Journal of Zhejiang Univearsity (Engineering Science), vol. 46, no. 10, pp. 1880-1886, 2012.

[15] W.-Q. Sun and X.-H. Huang, "Identification of vibration transfer path for the coupled system of water turbine generator set and power house," Journal of Vibration and Shock, vol. 33, no. 6, pp. 23-28, 2014.

[16] J. M. Nichols, M. Seaver, S. T. Trickey, L. W. Salvino, and D. L. Pecora, "Detecting impact damage in experimental composite structures: An information-theoretic approach," Smart Materials and Structures, vol. 15, no. 2, pp. 424-434, 2006.
[17] T. Schreiber, "Measuring information transfer," Physical Review Letters, vol. 85, no. 2, pp. 461-464, 2000.

[18] J. M. Nichols, "Examining structural dynamics using information flow," Probabilistic Engineering Mechanics, vol. 21, no. 4, pp. 420-433, 2006.

[19] J. M. Nichols, M. Seaver, S. T. Trickey, M. D. Todd, C. Olson, and L. Overbey, "Detecting nonlinearity in structural systems using the transfer entropy," Physical Review E: Statistical, Nonlinear, and Soft Matter Physics, vol. 72, no. 4, Article ID 046217, 2005.

[20] L. A. Overbey and M. D. Todd, "Effects of noise on transfer entropy estimation for damage detection," Mechanical Systems and Signal Processing, vol. 23, no. 7, pp. 2178-2191, 2009.

[21] D. Prichard and J. Theiler, "Generalized redundancies for time series analysis," Physica D: Nonlinear Phenomena, vol. 84, no. 3-4, pp. 476-493, 1995.

[22] D. Prichard and J. Theiler, "Generating surrogate data for time series with several simultaneously measured variables," Physical Review Letters, vol. 73, no. 7, pp. 951-954, 1994.

[23] J. Theiler, S. Eubank, A. Longtin, B. Galdrikian, and J. Doyne Farmer, "Testing for nonlinearity in time series: the method of surrogate data," Physica D: Nonlinear Phenomena, vol. 58, no. 92, pp. 77-94, 1992.

[24] T. Schreiber and A. Schmitz, "Improved surrogate data for nonlinearity tests," Physical Review Letters, vol. 77, no. 4, pp. 635-638, 1996. 


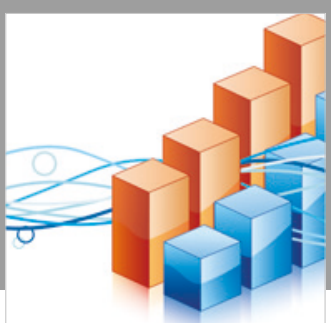

Advances in

Operations Research

\section{-n-m}
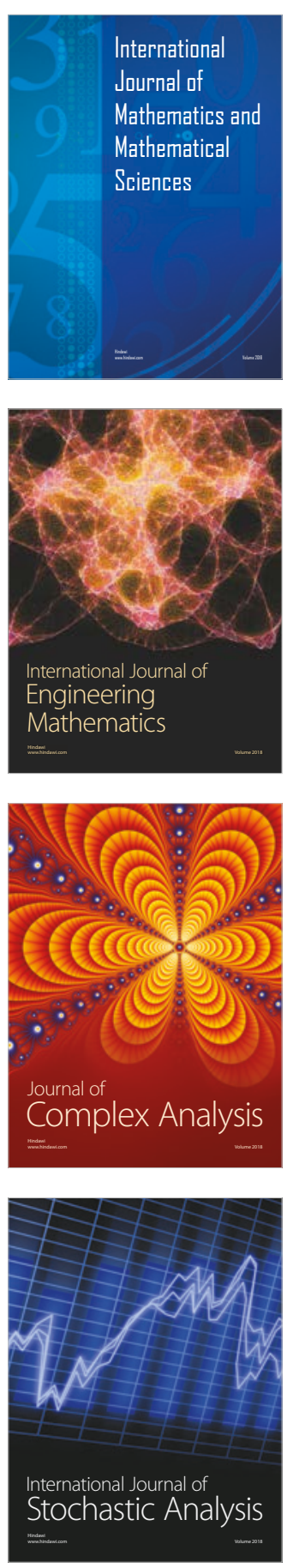
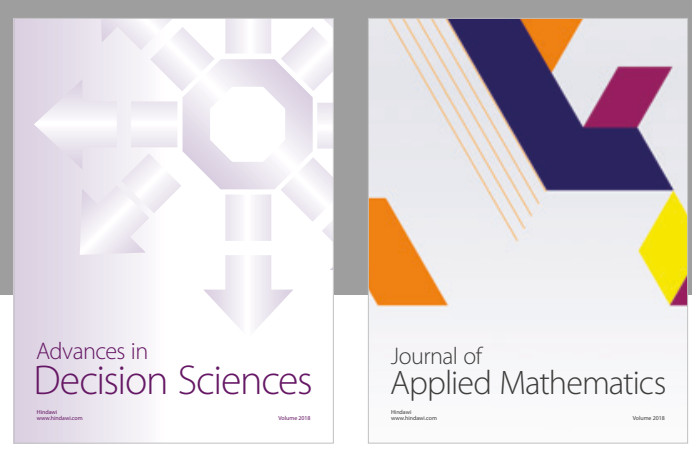

Journal of

Applied Mathematics
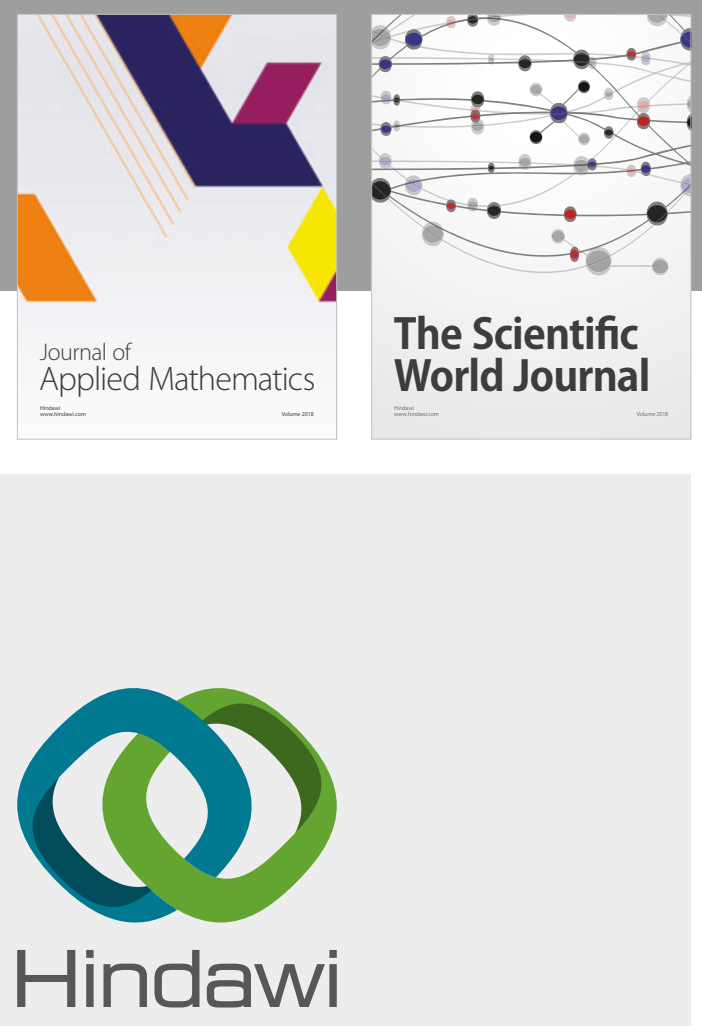

Submit your manuscripts at

www.hindawi.com

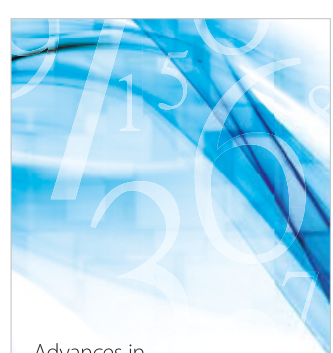

Advances in
Numerical Analysis
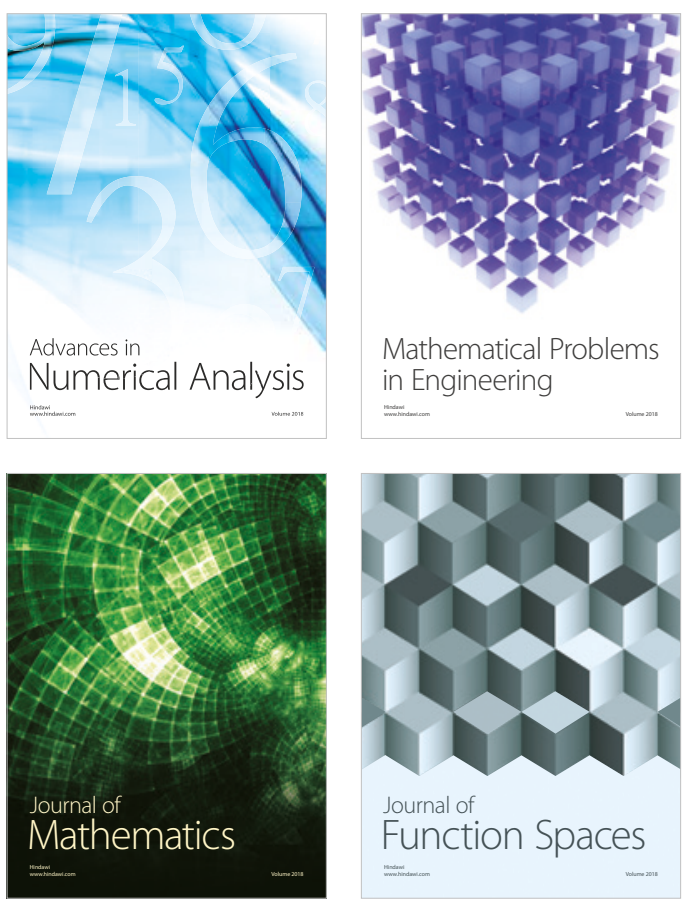

Mathematical Problems in Engineering

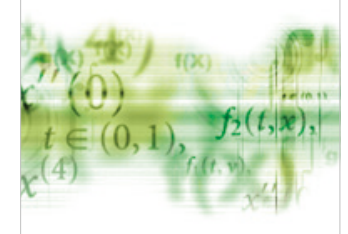

International Journal of

Differential Equations

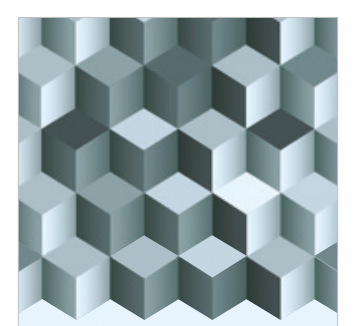

Journal of

Function Spaces

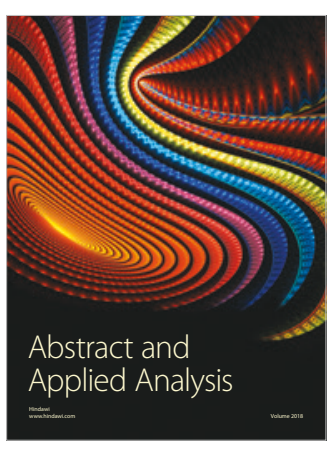

The Scientific

World Journal

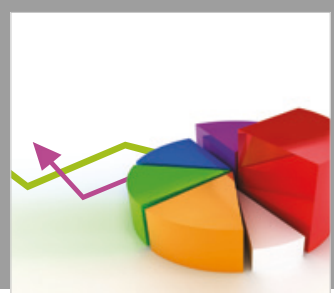

Journal of

Probability and Statistics
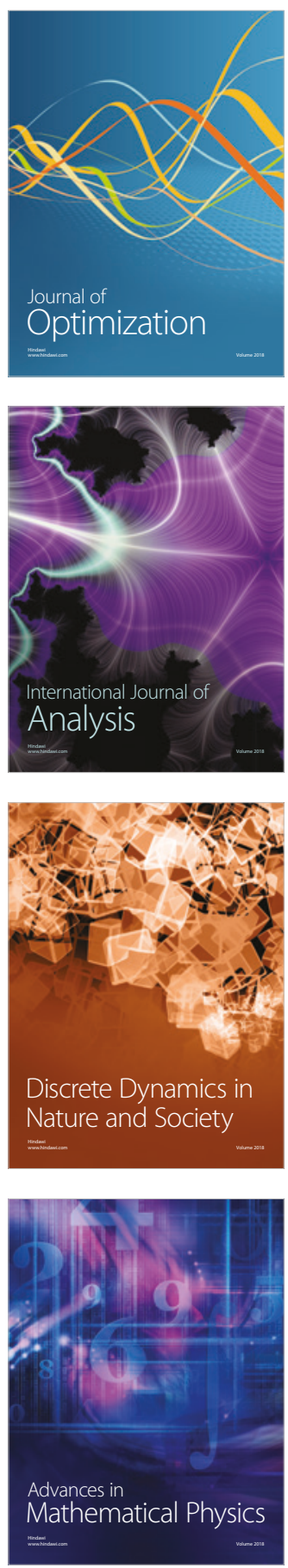